\title{
Um exemplo de neuropatia periférica que pode ser gerida pelo médico de família: neuropraxia do nervo peroneal
}

Patrícia Alexandra Cebola, ${ }^{1}$ Marta Filipa Rainho, ${ }^{2}$ António Alexandre Teixeira²

\section{RESUMO}

Introdução: A neuropraxia é uma forma de neuropatia periférica que o médico de família (MF) pode gerir por ter excelente prognóstico e reversibilidade total.

Descrição do Caso: Homem de 46 anos realizou colecistectomia por litíase biliar, em fevereiro de 2019, e por isso perdeu 10kg. No dia 18 de novembro de 2019 recorreu à consulta da MF por incapacidade de dorsiflexão do pé esquerdo, acompanhada de hipoestesia e diminuição da força muscular do membro inferior esquerdo desde o dia 11 de setembro de 2019, após estar sentado com a perna direita cruzada sobre a esquerda, durante cerca de três horas. Foi pedido eletromiograma, que demonstrou neuropraxia do nervo ciático popliteu externo esquerdo. Seis meses após a lesão inicial todos os sintomas desapareceram espontaneamente. Comentário: As manifestações clínicas da neuropraxia são bastante exuberantes e incapacitantes, o que pode levar o doente a pensar que tem uma doença grave. Saber abordar e explicar ao doente o que é esta lesão e o seu prognóstico é fundamental para evitar sobrediagnóstico e sobretratamento.

Palavras-chave: Neuropatia periférica; Nervo peroneal; Neuropraxia.

\section{INTRODUÇÃO}

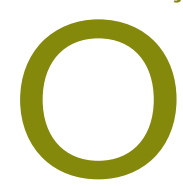

termo neuropatia periférica (NP) é aplicado a qualquer doença ou lesão que cause disfunção dos nervos periféricos. A sua prevalência é de $2,1 \%$, constituindo $8 \%$ dos motivos de consulta nos cuidados de saúde primários em indivíduos com mais de 55 anos. ${ }^{1}$ A sua incidência também é maior em jovens do sexo masculino ${ }^{2}$ e mais nos membros superiores. ${ }^{3}$

A neuropatia periférica pode-se manifestar de forma aguda ou crónica e os sinais e sintomas variam consoante o tipo de fibra afetada. O envolvimento de fibras sensoriais origina dormência, desequilíbrio, sensação de queimadura, prurido, formigueiro, picada ou dor. Envolvimento de fibras motoras pode originar fraqueza, atrofia muscular, alterações da marcha e quedas.

As NP podem ser classificadas consoante o número de nervos afetado. Se apenas um nervo está afetado de-

1. UCSP Amora, ACeS Almada-Seixal. Amora, Portugal.

2. USF Luisa Todi, ACeS Arrábida. Setúbal, Portugal. nomina-se mononeuropatia. Se mais do que um nervo está afetado, mas de forma assimétrica, denomina-se mononeuropatia múltipla (mais rara). Se mais do que um nervo está afetado e de forma simétrica denomina-se polineuropatia.

Neste artigo descreve-se um caso clínico como exemplo de mononeuropatia peroneal, que ilustra a prática de um médico de família (MF) que lida com a incerteza e que usa a semiologia, a história clínica, o exame objetivo e os conhecimentos de prevalência como ferramentas cruciais na prática clínica, para permitir o diagnóstico e o tratamento atempados do utente, fundamentais para que haja recuperação máxima da funcionalidade do nervo.

\section{DESCRIÇÃO DO CASO}

\section{Identificação e história pessoal}

Doente do sexo masculino, de 46 anos, leucodérmico, $73,5 \mathrm{~kg}$ de peso, 1,75m de altura, índice de massa corporal de $24 \mathrm{~kg} / \mathrm{m}^{2}$, com antecedentes pessoais de 
hipertensão arterial essencial desde fevereiro de 2019, sem evidência de lesão órgão-alvo; colecistectomia em fevereiro de 2019 por litíase biliar, que o fez perder 10kg. Sem hábitos tabágicos, alcoólicos ou outros toxicofílicos, sem alergias conhecidas. Tem todas as vacinas preconizadas pelo Programa Nacional deVacinação. Atualmente tem como medicação habitual lisinopril 20mg, que toma uma vez por dia.

\section{Caracterização sociofamiliar}

O utente tem uma licenciatura em engenharia eletrotécnica, está empregado, sendo diretor de obra e integra uma família nuclear de fase IV do ciclo de Duvall, classe II na escala de Graffar. É casado (a esposa tem 42 anos) e tem três filhos (dois rapazes de 13 e 9 anos e uma rapariga de um ano). Não apresenta problemas sociofamiliares relevantes.

\section{Antecedentes familiares}

Pai de 74 anos tem rinite alérgica, hipertensão arterial essencial e dislipidemia. Mãe de 75 anos tem rinite alérgica, hipertensão arterial essencial, dislipidemia e osteoartrose. Irmã de 42 anos tem rinite alérgica e hipertensão arterial essencial. Esposa e filhos têm rinite alérgica.

\section{História da doença atual}

No dia 11 de setembro de 2019, após estar sentado três horas, maioritariamente com a perna direita cruzada sobre a esquerda, levantou-se e sentiu hipoestesia acompanhada de diminuição da força muscular do membro inferior esquerdo. Encontrava-se ansioso, pois estava sentado na sala de espera do serviço de ginecologia-obstetrícia do hospital da residência a aguardar a resolução de uma complicação obstétrica da esposa. Após quatro horas todos os sintomas desapareceram, à exceção de incapacidade de realizar a dorsiflexão do pé esquerdo, o que tornava a marcha oscilante. Em 18 de novembro de 2019 recorreu à MF por persistência dos sintomas, não tendo feito qualquer medicação até à data da consulta.

Ao exame físico constatou-se marcha em steppage; prova de Lasègue negativa, compromisso total da dorsiflexão do pé esquerdo, diminuição da eversão do pé esquerdo e teste da sensibilidade dolorosa diminuído na face dorsal do pé esquerdo. Membro inferior direito não apresentava alterações. Durante o exame físico o utente não manifestou dor. Restante exame físico e neurológico sem alterações.

Atendendo às alterações observadas, e colocando como hipóteses diagnósticas radiculopatia compressiva por hérnia discal ou por neoplasia, ou neuropatia periférica, foram solicitados como exames complementares de diagnóstico (ECD), tomografia computorizada (TC) lombar, ecografia de partes moles da perna esquerda e estudo eletromiográfico (EMG) dos membros inferiores.

Em consulta de reavaliação do dia 28 de janeiro de 2020, referiu que já conseguia realizar parcialmente a dorsiflexão e totalmente a eversão do pé esquerdo. Também já não sentia hipoestesia, recuperações que se constataram em exame objetivo.

O utente disponibilizou os resultados dos exames pedidos em consulta anterior e observou-se que a TC lombar revelou discreta procidência difusa entre a $4^{\mathrm{a}} \mathrm{e}$ $5^{\mathrm{a}}$ vértebra lombar, moldando a vertente tecal anterior sem sinais de conflito de espaço radiculo-tecal. Procidência discal póstero-lateral entre a $5^{\mathrm{a}}$ vértebra lombar e $1^{\text {a }}$ vértebra sagrada, contactando a emergência radicular S1 esquerda. O EMG dos membros inferiores revelou neuropraxia recente do nervo ciático popliteu externo esquerdo. A ecografia de partes moles da perna esquerda não apresentou alterações significativas.

Atendendo ao diagnóstico de neuropraxia foi aconselhada a realização de fisioterapia.

Em consulta de reavaliação do dia 3 de junho de 2020 já se apresentava assintomático há três meses, conseguindo realizar todos os movimentos do pé esquerdo. Por isso, não realizou fisioterapia.

\section{COMENTÁRIO}

Nas mononeuropatias o nervo mais frequentemente afetado é o nervo mediano, que origina o síndroma do túnel cárpico. ${ }^{4}$ Apresenta incidência de 1-3 casos/ / 1.000 por ano e prevalência de $50 / 1.000 .^{5}$ Ao contrário da restante maioria das mononeuropatias afeta mais as mulheres. ${ }^{3,5}$ Quando há uma NP nos membros inferiores, o nervo ciático popliteu externo, também conhecido como peroneal comum ou fibular, é o mais afetado, tendo uma incidência de 17,2 por 100.000 e uma prevalência de 19 por 100.000 pessoas-ano. $^{3}$

A causa mais comum das mononeuropatias é o trauma, sendo que nas crianças é mais por fraturas e 


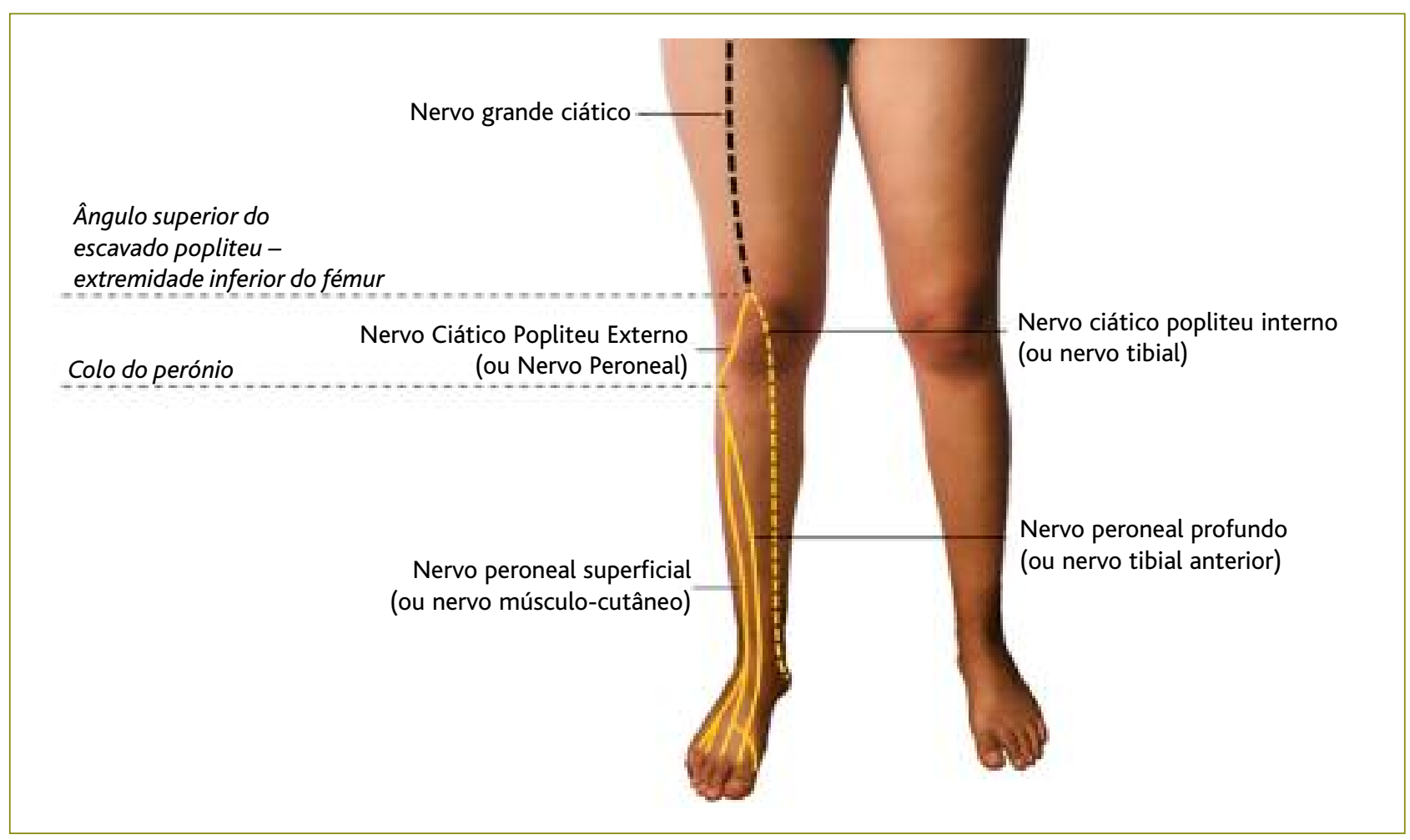

Figura 1. Representação gráfica simplificada do trajeto anatómico do nervo ciático popliteu externo (ou nervo peroneal). Destaque para a região do colo do perónio devido ao trajeto nervoso mais superficial a este nível.

lacerações, enquanto nos adultos acontece mais por aprisionamento do nervo ou compressão. ${ }^{6-7}$ Por outro lado, as principais causas de polineuropatia incluem diabetes, quimioterapia, vírus da imunodeficiência humana (HIV), doenças autoimunes, alcoolismo e doenças genéticas (doença de Charcot-Marie-Tooth, neuropatia sensorial hereditária).

A NP que resulta da lesão do nervo ciático popliteu externo denomina-se neuropatia peroneal e manifesta-se por incapacidade total ou parcial de dorsiflexão e/ou eversão do pé, acompanhada ou não de parestesias na face externa da perna e face dorsal do pé, poupando o $5^{\circ}$ dedo. A suscetibilidade para lesão deste nervo advém do seu trajeto anatómico (Figura 1).

O nervo grande ciático, ao chegar ao ângulo superior do escavado popliteu da extremidade inferior do fémur, bifurca-se, fornecendo os ramos ciático popliteu interno (ou tibial) e o ciático popliteu externo. Este último relaciona-se com o bordo interno do músculo bicípite crural, dirige-se obliquamente para baixo e para fora, alcança o colo do perónio, contorna-o num trajeto de cerca de $3 \mathrm{~cm}$, no compartimento externo da perna, internamente à inserção superior do músculo longo peroneal lateral. Aí divide-se nos seus ramos terminais, o nervo músculo-cutâneo ou peroneal superficial (responsável por inervar os músculos longo peroneal, curto peroneal, peroneal anterior e a pele da face dorsal do pé, exceto o $5^{\circ}$ dedo) e o nervo tibial anterior ou peroneal profundo (responsável por inervar os músculos tibial anterior, extensor comum dos dedos, extensor próprio do $1^{\circ}$ dedo do pé, peroneal anterior e pedioso).

Ao longo do seu trajeto, o nervo ciático popliteu externo também dá nervos colaterais que inervam as articulações do joelho, tíbio-társica superior, todas as articulações do pé (excepto as interfalângicas), pele da face antero-externa da perna e maléolo externo.

Os músculos inervados pelo nervo ciático popliteu externo participam em todos os movimentos do pé, mas fazem sobretudo a sua dorsiflexão. 
A porção do ciático popliteu externo que contorna o colo do perónio é muito superficial e encontra-se sobre a superfície óssea do perónio. Isto faz com que fique muito susceptível a traumas, sobretudo no contexto de luxação do joelho (onde ocorre lesão deste nervo em $40 \%$ dos casos); ${ }^{6-7}$ fratura da cabeça do perónio (onde ocorre lesão deste nervo em 1-2\% dos casos); ${ }^{8}$ lesões do músculo longo peroneal (por formação de fibrose que aprisiona o nervo supracitado) e compressões por tala de gesso, ligaduras, estar sentado com pernas cruzadas, imobilização prolongada ou por crescimento de tumores no nervo periférico.

Considerando a história clínica deste caso, assumese como causa mais provável de lesão do nervo ciático popliteu externo a permanência na posição de sentado com pernas cruzadas durante várias horas. É conhecido que a compressão por longos períodos de tempo feita ao nível do colo do perónio, com uma perna sobre a outra, já é suficiente para causar lesão no nervo ciático popliteu externo, sobretudo se a camada de tecido celular subcutâneo nesta zona for muito fina, como acontece nos indivíduos de baixo peso ou com grande emagrecimento num curto espaço de tempo. No caso descrito, o utente perdeu $10 \mathrm{~kg}$ recentemente, facto que apoia então a maior possibilidade de lesão desse nervo.

A neuropatia peroneal faz diagnóstico diferencial com lesão nas raízes lombo-sagradas que originam o nervo ciático (L4, L5, S1 e S2). No entanto, existem diferenças cruciais, quer na história clínica quer no exame objetivo, que nos permitem perceber qual a origem da lesão. A neuropatia peroneal manifesta-se por incapacidade total ou parcial de dorsiflexão do pé, acompanhada ou não de parestesias na face externa da perna e face dorsal do pé, poupando o $5^{\circ}$ dedo. Já a incapacidade de dorsiflexão do pé resultante de radiculopatia lombo-sagrada é acompanhada de dor que começa por ser do tipo mecânica sem irradiação, mas que à medida que a patologia de base vai progredindo, vai comprimindo o nervo ciático e desencadeando irradiação e alterações sensitivas, descritas pelo doente de maneira diferente, consoante o nível de compressão na coluna. Se for ao nível de L4, as queixas são sentidas na face antero-externa da coxa, face interna da perna, bordo interno e $1^{\circ}$ dedo do pé. Se for em L5 são sentidas na face externa da coxa e perna e face dorsal do pé, poupando o $1^{\circ} \mathrm{e} 5^{\circ}$ dedos. Se for em S1 é ao nível da face posterior e média da coxa, face posterior e lateral da perna, bordo externo e $5^{\circ}$ dedo do pé. Se for ao nível de S2, as queixas são sentidas na face póstero-interna da coxa e perna.

Ao exame objetivo, a primeira prova que permite distinguir entre lesão lombar e peroneal é a prova de $L a$ sègue, que é positiva apenas em lesões lombares. Seguidamente, enquanto numa lesão a nível do nervo ciático popliteu externo os reflexos osteotendinosos estão mantidos, no caso de uma radiculopatia lombo-sagrada eles estão diminuídos, consoante a raíz afetada. Numa lesão em L4 está diminuído o reflexo rotuliano, numa lesão em L5 está afetado o reflexo de Hamstring ou isquiotibial e numa lesão em S1 está afetado o reflexo aquiliano.

Já ao nível dos movimentos do pé, outro pormenor que permite distinguir entre lesão peroneal de lesão em L5 é que a incapacidade de realizar dorsiflexão do pé por lesão peroneal é acompanhada por incapacidade de eversão do pé. No caso de lesão em L5, a incapacidade de dorsiflexão é acompanhada de incapacidade de inversão do pé. Outro sinal sensível para lesão peroneal é o facto de que fazer contra resistência na dorsiflexão do pé proporciona dor ao nível da região do trajeto do nervo ciático popliteu externo.

Considera-se o ECD de eleição o EMG, pois, ao estudar as variações da atividade elétrica das unidades motoras em repouso, durante a contração voluntária e em resposta a estimulação elétrica, o EMG permite estabelecer a localização, tipo de lesão e seguimento do processo de regeneração.

Nas NP, o tipo de lesão é descrito com base na escala de Seddon, ${ }^{9}$ construída, em 1942, pelo ortopedista Sir Herbert Seddon. Nesta escala as lesões são descritas como neuropraxia, axonotmese e neurotmese. As diferenças entre elas baseiam-se fundamentalmente no grau de reversibilidade. ${ }^{10}$

Na neuropraxia regista-se perda motora e/ou sensitiva por perda temporária de mielina, mas mantém-se a integridade do nervo. A condução nervosa após lesão mantém-se mais lenta, recuperando gradualmente a sua velocidade normal à medida que ocorre regeneração da mielina. A função nervosa é recuperada integralmente, sem necessidade de tratamento. ${ }^{11-12}$

$\mathrm{Na}$ axonotmese, quer a mielina quer o axónio estão lesados, mas o estroma de suporte ao neurónio 
(células de Schwann, endoneuro e perineuro) permanece intacto e por isso ocorre a criação de novos ramos nervosos distais à lesão e regeneração dos axónios. $\mathrm{O}$ prognóstico vai depender do grau de desorganização neuronal interna, da distância ao órgão-alvo, das particularidades anatómicas e da complexidade do nervo (nervos com menos ramificações e só com função motora ou sensitiva têm maior potencial de recuperação). De uma forma geral, a recuperação é esperada, mas de forma mais lenta do que na neuropraxia. Nestes casos, é benéfico recorrer à fisioterapia, pois permite controlar a dor e eventual edema, manter ou aumentar a amplitude de movimentos e prevenir contraturas de tecidos moles. ${ }^{11-12}$

Na neurotmese, o nervo e tecido circundante são completamente lesados, não sendo possível recuperar a função nervosa se não houver atempadamente cirurgia de reconstrução, com eventual resseção de cicatriz intraneural e reparação com enxerto nervoso. ${ }^{11-12}$

O EMG deve ser realizado idealmente entre sete e dez dias desde o início dos sintomas, pois se houver degeneração axonal distal à lesão, consequente da separação entre axónio e corpo neuronal (a chamada degeneração walleriana), esta só se inicia ao $3^{\circ}$ dia após a lesão e é completada entre o $5^{\circ}$ e $8^{\circ}$ dia para fibras motoras e entre o $7^{\circ}$ e $10^{\circ}$ dia para fibras sensitivas. Caso o utente não esteja a recuperar dos sintomas de neuropraxia ou o exame tenha sido inconclusivo deve-se repetir o exame a partir das três semanas pós-lesão, pois só a partir dessa altura é evidente a não inervação muscular e permite diagnosticar com maior acuidade os graus de axonotmese e neurotmese. ${ }^{13-14}$

Também é importante a informação dada por um exame de imagem, pois não só permite perceber qual a causa da lesão nervosa, como também dar mais informações sobre a extensão e a gravidade da lesão. Conseguir-se-á, assim, definir o melhor tratamento possível.

No caso do utente descrito, o tipo de lesão do nervo ciático popliteu externo foi uma neuropraxia e, por isso, o utente foi recuperando gradualmente as capacidades perdidas no espaço de seis meses, sem ser necessário recorrer a qualquer terapêutica. Pelos sinais detetados em exame objetivo, uma das hipóteses de diagnóstico levantadas foi o síndroma compressivo a nível lombar. Portanto, foi pedida, numa primeira abordagem, TC lombar. No entanto, como a história clínica era muito sugestiva de lesão traumática na perna, foi pedido o eletromiograma para esclarecer melhor a localização da lesão.

Por estes ECD, o utente demonstrou ter lesão no nervo ciático popliteu externo e hérnia discal entre a $5^{\mathrm{a}}$ vértebra lombar e a $1^{\text {a }}$ vértebra sagrada na TC lombar, podendo ambas ser responsáveis pelas manifestações clínicas do caso. No entanto, o utente não referia lombalgia e, embora tivesse referido que sentia parestesias em toda a perna, não sentia na coxa, o que não está de acordo com a alteração de sensibilidade que era suposto sentir pela sua hérnia.

Como a causa da neuropraxia parecia ser trauma das estruturas adjacentes ao nervo ciático popliteu externo e como o utente foi melhorando em termos de sintomatologia, uma ecografia das partes moles pareceu ser suficiente como primeiro exame de imagem.

Analisando a marcha diagnóstica realizada neste caso clínico, para torná-la mais eficiente e efetiva, poderia ter sido pedido inicialmente apenas o EMG, pois tem capacidade para indicar a localização e o grau da lesão. Depois, consoante os resultados obtidos em EMG, poderia pedir-se um exame de imagem. A escolha de qual o exame imagiológico a pedir iria depender do grau da lesão, causa e tratamento a aplicar (cirúrgico ou conservador).

Em relação a tratamento farmacológico optou-se por não introduzir terapêutica dirigida às queixas neuropáticas, pois o utente foi melhorando progressivamente ao longo das reavaliações. No entanto, considera-se que poderia ter sido introduzido numa fase inicial para averiguar se as queixas se atenuariam mais depressa.

O utente do caso clínico entendeu a causa da sua doença e o seu prognóstico, pois, ao longo das consultas, houve um esforço por parte da MF para construir uma relação de empatia e confiança com o utente. Estabeleceu-se uma comunicação cuidada e clara, em que se transmitiu conhecimento, preocupação e interesse na saúde do utente e no seu meio envolvente.

Em conclusão, um caso de neuropraxia, embora seja pouco frequente na prática clínica de um MF, é importante saber abordá-lo, pois sendo um grau de lesão de nervo periférico ligeiro e reversível as suas manifestações clínicas são bastante exuberantes e incapacitantes, o que pode levar a que o doente tenha a 
expectativa de ter algo muito grave. Fazer uma história clínica rigorosa, pedir os ECD necessários, criar uma relação de confiança com o utente e saber explicar o que é esta lesão e o seu prognóstico é fundamental para evitar sobrediagnóstico e sobretratamento.

\section{REFERÊNCIAS BIBLIOGRÁFICAS}

1. Azhary H, Farooq MU, Bhanushali M, Majid A, Kassab MY. Peripheral neuropathy: differential diagnosis and management. Am Fam Physician. 2010;81(7):887-92.

2. Cheng YJ, Gregg EW, Kahn HS, Williams DE, De Rekeneire N, Narayan KM. Peripheral insensate neuropathy: a tall problem for US adults? Am J Epidemiol. 2006;164(9):873-80.

3. Hanewinckel R, Ikram MA, Van Doorn PA. Peripheral neuropathies. Handb Clin Neurol. 2016;138:263-82.

4. Atroshi I, Gummesson C, Johnsson R, Ornstein E, Ranstam J, Rosén I. Prevalence of carpal tunnel syndrome in a general population. JAMA. 1999;282(2):153-8.

5. De Krom MC, Knipschild PG, Kester AD, Thijs CT, Boekkooi PF, Spaans F. Carpal tunnel syndrome: prevalence in the general population. J Clin Epidemiol. 1992;45(4):373-6.

6. LaPrade RF, Terry GC. Injuries to the posterolateral aspect of the knee: association of anatomic injury patterns with clinical instability. Am J Sports Med. 1997;25(4):433-8.

7. Siqueira R. Lesões nervosas periféricas: uma revisão [Peripheral nerve injury: a review]. Rev Neurocienc. 2007;15(3):226-33. Portuguese
8. Noble J, Munro CA, Prasad VS, Midha R. Analysis of upper and lower extremity peripheral nerve injuries in a population of patients with multiple injuries. J Trauma. 1998;45(1):116-22.

9. Seddon HJ. A classification of nerve injuries. Br Med J. 1942;2(4260): 237-9.

10. Gilliatt RW, Hjorth RJ. Nerve conduction during Wallerian degeneration in the baboon. J Neurol Neurosurg Psychiatry. 1972;35(3):335-41.

11. Campbell WW. Evaluation and management of peripheral nerve injury. Clin Neurophysiol. 2008;119(9):1951-65.

12. Poage $C$, Roth $C$, Scott B. Peroneal nerve palsy: evaluation and management. J Am Acad Orthop Surg. 2016;24(1):1-10.

13. Chaudhry V, Cornblath DR. Wallerian degeneration in human nerves: serial electrophysiological studies. Muscle Nerve. 1992;15(6):687-93.

14. Bergquist ER, Hammert WC. Timing and appropriate use of electrodiagnostic studies. Hand Clin. 2013;29(3):363-70.

\section{CONFLITO DE INTERESSES}

Os autores declaram não possuir quaisquer conflitos de interesse.

\section{ENDEREÇO PARA CORRESPONDÊNCIA}

Patrícia Alexandra Cebola

E-mail: pcebola@campus.ul.pt

https://orcid.org/0000-0002-7033-1644

Recebido em 08-12-2020

Aceite para publicação em 29-07-2021

\section{ABSTRACT}

\section{AN EXAMPLE OF PERIPHERAL NEUROPATHY THAT CAN BE MANAGED BY THE GENERAL PRACTITIONER: PERONEAL NERVE NEUROPRAXIA}

Introduction: Neuropraxia is a rare form of peripheral nerve injury. All general practitioners (GP) can manage this condition due to its excellent prognosis and complete reversibility.

Case Description: 46-year-old male, had cholecystectomy for gallstones in February 2019, having lost $10 \mathrm{~kg}$. On the 18th of November 2019, he presented to his GP with an inability to lift his left foot, accompanied by hypoesthesia and decreased strength in the entire left lower limb since the 11th of September 2019, after being seated for three hours, with his right leg crossed over the left. The GP requested an electromyogram test, which revealed neuropraxia of the left peroneal nerve. All symptoms disappeared spontaneously six months after the initial injury.

Comment: Although reversible, neuropraxia has a very exuberant clinical presentation and can lead the patient to think that suffers from a serious illness. Knowing how to approach and make sure that the patient understands the disease and its prognosis is essential to avoid overdiagnosis and overtreatment.

Keywords: Peripheral neuropathy; Peroneal nerve; Neuropraxia. 